\title{
Scale-Free based network structure for Data center
}

\author{
Lyu, $\mathrm{Qi}^{1, \mathrm{a}}$ and Cheng, Daoxu $\mathrm{u}^{2, \mathrm{~b}}$ \\ ${ }^{1}$ School of Nanjing University, Nanjing 210093, China; \\ 2 School of Nanjing University, Nanjing 210093, China. \\ alvsoft@gmail.com, bcdx@nju.edu.cn
}

Keywords: data center, laaS, network structure, big data, scale free.

\begin{abstract}
The idea of big data processing is widely accepted nowadays, which makes it critical for IaaS to support network infrastructures in large scale. This paper presents a novel network model based on scale free network, which explored the influence to scale free property with a maximum degree constraint, and detailed deployment node structures under different balance factor. This paper analyzed the theoretical nature of scale free combined with data center application, presents the simulation results and the verification at large scale for various configurations.
\end{abstract}

\section{Introduction}

The impact of big data driven the application of large scale of servers, which trigger the requirement of data center construction as a support infrastructure. Unfortunately, traditional network architecture of data center is expensive and lack the high availability, flexibility and scalability properties which are essential for big data applications.

Scale free network is a complex network form between structure network and random network, which has key characters of growth and preferential attachment [1]. Its distribution of degree follows power law with the factor of $3[2,3]$, which is suitable for construction of networks of data center.

There's lots of research over scale free networks: Dorogovtsev et al. [4] and Krapivsky et al. [5] analyzed the proper of BA model. Albert and Barabási presented a modificated BA algorithm [6], generating scale-free network which would follow the power law distribution with desired $\gamma$ value. With the in-depth study of scale-free networks, Goh et al. concluded there's parameters other than the degree of network concentration, like BC (betweenness centrality), which is first be quantizated by Anthonisse [9] and Freeman [10]. Goh et al. discovered the distribution of BC value also follows similar power low characters:

$$
P(\ell) \approx \ell^{-\delta}
$$

And Goh et al. concludes, the value of $\delta$ is approximately constant and equals to 2.2 in several examples of practice world. In the following researches [11], Goh et al. discovered $\delta \approx 2.2$ in biological protein structure, biological metabolic networks and co-author relationships. However, $\delta \approx 2.0$ in WWW. Solé et al. and Vázquez et al. discovered [12, 13], compared with small-world network, scale-free network has different characters in extremely few nodes with extremely aggregation node, and extremely low of even distance as $\log \log n$, which is valuable, worthy of study and research in data center networks.

\section{DATA CENTER ORIENTED SCALE- FREE NETWORK}

Analysis of scale-free network in data center. The ultra-low distance character of scale-free network decrease the transition duration of datagram. However, high clustering properties is also required in data center network due to high loads. Although the clustering coefficient decrease along with the increasing of average degree distance. There also have researches of generating scale-free networks with specified clustering coefficient [14]. Generally speaking, the properties of low distance with high clustering of scale-free network, meets the needs of the data center networks. 
The scale-free networks is mapped to a specific data center network architecture. László Gyarmati et al.[15], presented a improved BA algorithm called Scafida, which try to meeting the need of data center network with scale-free networks. The approach introduced the concept of heterogeneous networks, ie all nodes in the network are not entirely consistent, the interface number of server or switches can be differs, by record the information of total nodes list and assigned nodes, it'll construction a scale-free networks under the constraint of node and switch resources. Thereby generate a practicable network structure.

As László Gyarmati believes, such of constraint will not paralyze scale free property by a simulated experience result. However, a further in-depth analysis indicates that viewpoint may have some following problems:

1) Any method which introduce constraint of maximum degree, will eventually block the properties of scale-free networks.

2) The Scafida was a two-stage approaches, which generate network structure first, than assign roles for nodes. Which lack the consideration of complex situations like switches form a loop.

3) The approach of strict restrict node/switch resources during construction, essentially reversed cause and effect.

The final object is not to use as much as possible of all network equipment resources, but to connect all the nodes reasonable and effect. In fact the number of network devices can be better optimized from exhaustively used.

In the following sections, this paper try to give a different theoretical models for general approach of data center network construction by analyze first question. Than analyze and explain the other two questions.

Scale-free network and maximum degree. By the nature of scale-free networks, the distribution of node degree of compliance with power-law distribution, which is $P(d=k) \propto k^{-r}$.

For a scale-free network with node $n$, the expectation of maximum degree is $\operatorname{MaxDegree}(n)=c((n-1) / \gamma)^{1 /(\gamma-1)}$

Clearly, there is a limit as the maximum degree, assuming $\operatorname{MaxDegree}(n)=k$, then when the network scale growth to $n=\left(c \gamma(k / c)^{\gamma}+k\right) / k$, will not maintain scale-free networks property any more.

Thus, by a given maximum degree constraint, network will no long maintain scale-free property after a certain size.

Scale-free construction algorithm with maximum degree constraints. While we can't construct scale-free networks under a constraints of the maximum degree, it is still possible to achieve a certain degree of approximation by the idea of divide and conquer.

Assume that node is limited to maximum of $k$, and construct a $n$ scale-free network. Step.1, generate network $G_{0}$ by BA algorithm with parameter of $m=1$. For $G_{0}$, for any node x, if Degree $(x)>k$, then extract all nodes connected with $x$, and generate network $G_{1}$ by BA algorithm with node size $N_{1}=\operatorname{Degree}(x)+1$. The $G_{1}$ is regarded as a logical node in $G_{0}$, which has maximum degree as $\sum_{y \in G_{1}} k$-Degree $(y)$, which will replace $x$ and all nodes connected with in $G_{0}$. Keep iterate above process until all nodes in $G_{0}$ follow the constraint of maximum degree. 


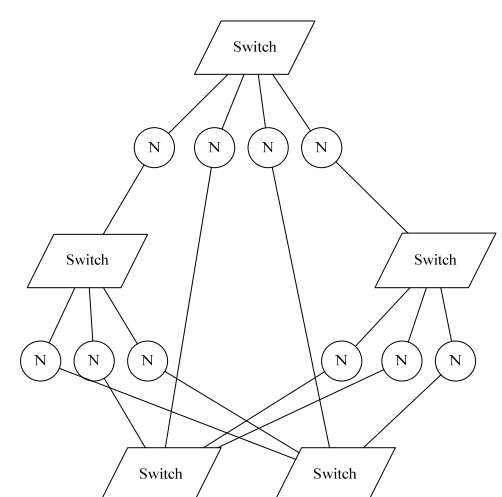

Fig. 1 Balance of S and N

\section{ADJUSTMENT OF BALANCE FACTOR}

Balance factor can be introduced by the proportion of $N-N$ (Node-Node connection schema) and $\mathrm{N}$-S (Node-Switch connection schema), which can be defined as balance factor:

Nodes in network follows a typical schema of $N$-S, which is every nodes will be connected by switch. Assuming $N-S$ is the only connection mode, then the number of $S$ will be a fixed value of $w$.

Let $w+q$ be the number of $S$ in the generated network. Then $q$ is the balance factor, ranging from $[-w, \infty]$

Balance factor describes the usage of network devices, which will impact network performance and construction cost greatly. This section, we will analysis the optimal value of the balance factor.

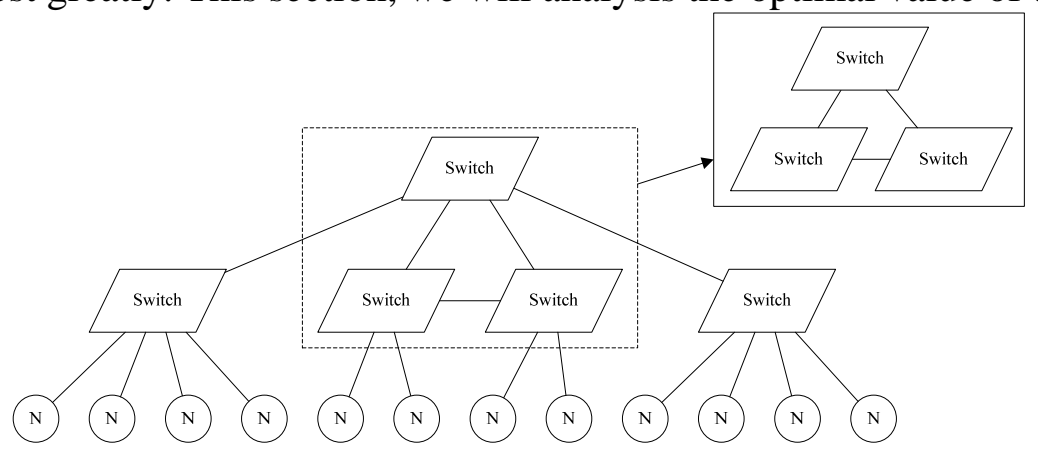

Fig. 2 Excessive Switches

For q=0. We'll analyze the most common case: $q=0$. Let's assume that $n \times t=k \times w$, to ensure $N$-S schema is the only form in network. For cases like $n \times t \neq k \times w$, there'll be a certain probability of occurrence of $S$ - $S$ schema, which can be simplified for the ease of analysis. Thus, each $N$ nodes connected with $S$ nodes. From the perspective of resource utilization, the connectivity of $n$ nodes will meet total port of $w$ switches exactly, which intuitively also to be a perfect connection schema. As showed in Fig. 1.

For q>0 . Since the reduce of $N$ nodes, there'll be lots of $S$-S connection schema to consume internal interconnection interfaces of $S$ switches. For $S$ - $S$ mode, there'll be a requirement for network equipment to avoid loops. Otherwise, if multiple switches cascaded and formed a cyclic loop, there'll be broadcast storm which will severely weakened switch performance. As showed in Fig. 2.

By span tree algorithm, the network can be simplified to tree, which can eliminate loops and make the network structure usable in ordinary switches. However, this approach is equivalent to deleted some part of $S$-S links. And lack the ability to dynamic routing. The few remaining $S$-S forms span tree linked as daisy chain, which provide relatively small bandwidth.

Thus, otherwise the network equipments are smart devices which capable of form dynamically span trees, the network performance will not increase while increase more network switches.

On the other hand, with the further increasing of $q, S-S$ schema can no longer consume internal interconnection interface of $S$ switches. There'll be less $N$ nodes for each $S$ switch, and $N-S-N$ schema 
begin to emerge. It is obviously $N-S-N$ is equivalent to $N-N$, which indicates the increasing if $q$ will come to a limitation naturally.

For $\mathbf{q}<\mathbf{0}$. There'll be more $N-N$ connection pattern due to the reduce of $\mathrm{S}$ nodes. Consider that the relatively small number of interfaces for $N$ nodes. For a value of $t=2$, a large number of $N-N$ schema will eventually form one straight line connection. For other values like $t=3$; $t=4$, a large number of $N-N$ would constitute a cellular link, and mesh link. As showed in Fig. 3.

Compared with the connectivity of switch based networks, $N-N$ schema will weaken the network in performance and fault tolerance.

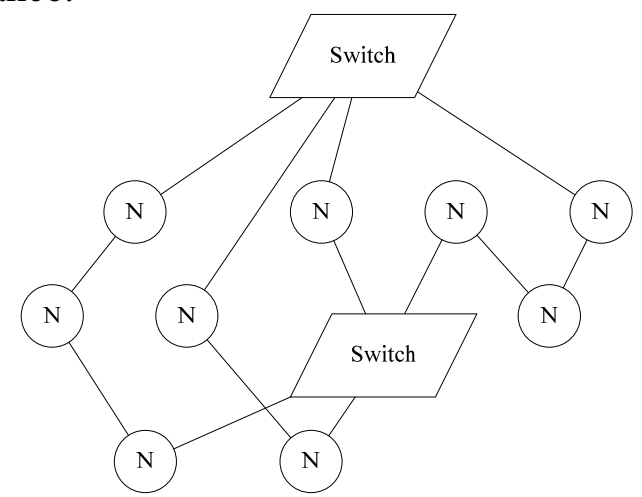

Fig. 3 Insufficient Switches

However, from the point of resource consumption view, minor $N-N$ node is beneficial for network. For switches will essentially allow dynamically connections between all links, while for scale-free network, not all nodes are needed to interconnect through switches. As a result, only partial links are dynamically selected, which is possible for some links form as static P2P $N-N$ schema without harm the network performance.

After introduced balanced factor, the construct progress can be described as follow steps:

1. Determine node numbers of $S$ and $N$, and the upper limit of the connections per node.

2. Select a node pair from $S$ and $N$ randomly to establish a connection, which forms the initial graph.

3. Enter main loop, select a node $a$ from node group established connections, which based on the weight of node degree. Ensure that the probably of selection is in proportion to its degree.

4. Determine the allowed connectivity schemas, based on the type of currently selected node( $S$ or $N$ ), and also consider balance factor. Then select a node $b$ from the remaining nodes randomly which is not connected yet, to established a connection pair between $a$ and $b$.

5. Loop above steps until all the nodes have established at least one connection. The construction algorithm ends.

\section{ANALYSIS AND SIMULATION}

Average node distance. For a purpose of simplicity and without lose of generalization, let's assume all nodes' interface number is $t$ and all switches' interface number is $k$.

Thus, the average route distance:

$$
D=\log n / \log k(t-1) \times(1+q / w)
$$

The above formula only established when no $N-S-N$ schema exist in network. When $q$ keep increasing until $N-S-N$ appears, the average distance of $D$ will not change obviously. Since the larger $q$ will consume more switches without significantly performance gain, which can be excluded from discussion. In this case, the proper value of $q$ in formula, can ranged in approximately[-0.1..0.5]. 


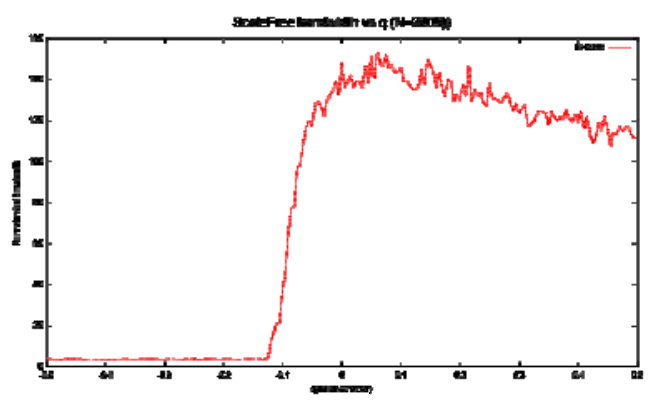

Fig. 4 Network Performance vs $q$ Factor

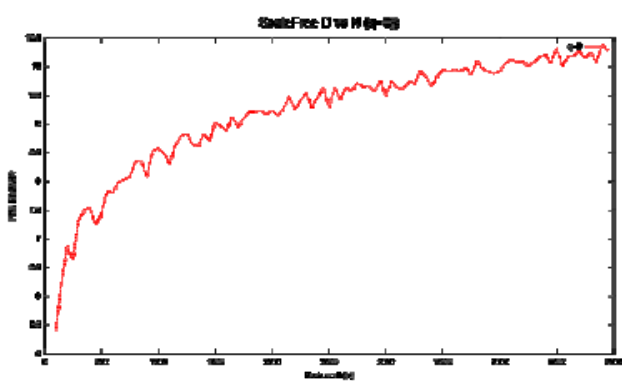

Fig. 5 Average Route Distance vs Network Scale $\mathrm{N}$

Fig.4 indicates the network performance with various value of $q$. As the q slightly lower the pointer of $q=0$, the network performance still holds. However, the performance failed rapidly while the value $q$ keep decreasing. On the other side, raising $q$ won't increase network performance, and even decrease when $q$ increased too much.

Fig.5 shows the relationship of average routing hops with network scale of $N$. Due to the usage of tiny switches $(k=8)$, the average route hops maintained at a relatively high level (10 for 5000 nodes) with the increasing of network scale. But despite the linear increasing of $N$, the increment of average route hops begin to drop, which indicate the character of scale free.

Average route hops. Fig. 6 shows the change of switch ports( $k$ ) with average distance of networks. Obviously more interfaces of switches will form a smaller average distance. But the gap between $k=8$ and $k=48$ is not large.

Overall network bandwidth. Fig.7 indicates impact of various number of switch interface $k$. Although we can see a different from various $k$ values, which shows overall bandwidth will be a bit better by using higher $k$ switches. However the different is not obvious. Due to the long tail effect of scale-free networks, a few nodes which has high connectivity contribute great in route distance, but only 1 in bandwidth. Furthermore, since the a long tail effect, most nodes still have a degree less than 8 even under the $k=8$ configurations. Thus, the gap is minor with different configurations of $k$.
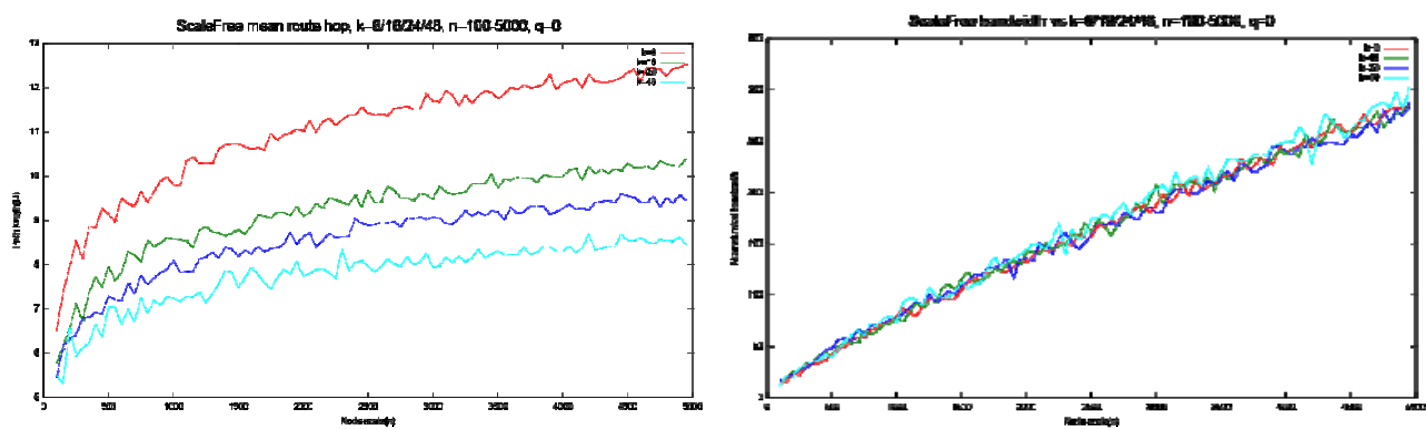

Fig. 6 Average Route Distance vs $k$ Fig. 7 Network Bandwidth vs $k$

\section{Conclusion}

This paper analyzes the theoretical aspects of appliance scale-free networks with data center network. Analysis the property of BA constructed network with maximum degree constraint, which described in Scafida [15]. We also presents multi-layer construction method using an recursive idea of divide and conquer. And analyzed the clustering properties, various type of node connection forms, and the impact on network performance. 


\section{References}

[1] R. K. Merton, The matthew effect in science, Science, vol. 159, no. 3810, pp. 56-63, 1968.

[2] D. Price, Statistical studies of networks of scientific papers, in Statis-tical Association Methods for Mechanized Documentation: Symposium Proceedings, vol. 269. US Government Printing Office, 1965, p. 187.

[3] S. Redner, How popular is your paper? an empirical study of the citation distribution, The European Physical Journal B-Condensed Matter and Complex Systems, vol. 4, no. 2, pp. 131-134, 1998.

[4] S. N. Dorogovtsev, J. F. F. Mendes, and A. N. Samukhin, Structure of growing networks with preferential linking, Physical Review Letters, vol. 85, no. 21, p. 4633, 2000.

[5] P. L. Krapivsky, S. Redner, and F. Leyvraz, Connectivity of growing random networks, Physical review letters, vol. 85, no. 21, p. 4629, 2000.

[6] A.-L. Barab ' asi, R. Albert, and H. Jeong, Scale-free characteristics of random networks: the topology of the world-wide web, Physica A: Statistical Mechanics and its Applications, vol. 281, no. 1, pp. 69-77, 2000.

[7] G. Bianconi and A.-L. Barab ' asi, Competition and multiscaling in evolving networks, EPL (Europhysics Letters), vol. 54, no. 4, p. 436, 2001.

[8] L. A. Adamic and B. A. Huberman, Power-law distribution of the world wide web, Science, vol. 287, no. 5461, pp. 2115-2115, 2000.

[9] J. M. Anthonisse, The rush in a graph, Amsterdam: University of Amsterdam Mathematical Centre, 1971.

[10] L. C. Freeman, A set of measures of centrality based on betweenness, Sociometry, pp. 35-41, 1977.

[11] K.-I. Goh, E. Oh, H. Jeong, B. Kahng, and D. Kim, Classification of scale-free networks, Proceedings of the National Academy of Sciences, vol. 99, no. 20, pp. 12 583-12588, 2002.

[12] R. V. Sol ' e, R. Pastor-Satorras, E. Smith, and T. B. Kepler, A model of large-scale proteome evolution, Advances in Complex Systems, vol. 5, no. 01, pp. 43-54, 2002.

[13] A. V' azquez, A. Flammini, A. Maritan, and A. Vespignani, Modeling of protein interaction networks, Complexus, vol. 1, no. 1, pp. 38-44, 2002.

[14] C. Herrera and P. J. Zufiria, Generating scale-free networks with adjustable clustering coefficient via random walks, in Network Science Workshop (NSW), 2011 IEEE. IEEE, 2011, pp. 167-172.

[15] L. Gyarmati and T. Trinh, Scafida: A scale-free network inspired data center architecture, ACM SIGCOMM Computer Communication Review, vol. 40, no. 5, pp. 4-12, 2010. 